\title{
The Adomian Decomposition Method for Solving Volterra-Fredholm Integral Equation Using Maple
}

\author{
Hunida M. Malaikah \\ Department of Mathematics, Faculty of Science, King Abdulaziz University, Jeddah, KSA \\ Email: hmalaikah@kau.edu.sa
}

How to cite this paper: Malaikah, H.M. (2020) The Adomian Decomposition Method for Solving Volterra-Fredholm Integral Equation Using Maple. Applied Mathematics, 11, 779-787.

https://doi.org/10.4236/am.2020.118052

Received: July 19, 2020

Accepted: August 24, 2020

Published: August 27, 2020

Copyright ( 2020 by author(s) and Scientific Research Publishing Inc. This work is licensed under the Creative Commons Attribution International License (CC BY 4.0).

http://creativecommons.org/licenses/by/4.0/

\section{(c) (i) Open Access}

\begin{abstract}
In this paper, Adomian decomposition method (ADM) is used to solve the Volterra-Fredholm integral equation. A number of examples have been presented to explain the numerical results, which is the comparison between the exact solution and the numerical solution, and it is found through the tables and the amount of error between the exact solution and the numerical solution, it is very small and almost non-existent and is also illustrated through the graph how the exact solution of completely applies to the numerical solution This proves the accuracy of the method, which is the Adomian decomposition method (ADM) for solving the Volterra Fredholm integral equation using Maple 18. And that this method is characterized by ease, speed and great accuracy in obtaining numerical results.
\end{abstract}

\section{Keywords}

Volterra-Fredholm Integral Equation, Adomian Decomposition Method, Maple18

\section{Introduction}

The current research intends to the Adomian decomposition method for solving Volterra-Fredholm integral equation using Maple18.

Integral equations are the basic sciences in our real life, and they explain physical, chemical, engineering, and medical phenomena, and more than that, they contribute greatly to reaching analytical and numerical solutions to these phenomena in various areas of our lives [1] [2]. There are several studies of Adomian decomposition metod, convergence and accuracy of Adomian's decomposition method for the solution of Lorenz equationsis studied in [3]. Solv- 
ing Riccati differential equation using Adomian's decomposition method is given in [4]. An adaptation of Adomian decomposition for numeric-analytic integration of strongly nonlinear and chaotic oscillatorsis studied in [5]. The extended Adomian decomposition method for fourth order boundary value problems is given in [6]. The use of the Adomian decomposition method for solving multipoint boundary value problems is mentioned in [7]. [8] developed a new algorithm for evaluating Adomian polynomials. [9] studied an efficient algorithm for the multivariable Adomian polynomials. [10] found convenient analytic recurrence algorithms for the Adomian polynomials. A review of the Adomian decomposition method and its applications to fractional differential equations is given in [11]. [12] covers a bibliography of the theory and applications of the Adomian decomposition method. We find that solutions of nonlinear integral equations are more difficult to solve than linear integral equations and there are many analytical and numerical methods for solving linear and nonlinear integral equations mentioned in the references [13] [14] [15] [16]. We discuss the numerical solution of the integral Volterra equation of the second type using an implicit trapezoidal [17] [18]. The Adomian decomposition method of the Fredholm integral equation of the second kind using MATLAB and Maple is demonstrated in [19]. The Adomian decomposition method was applied to solve the Fredholm integral equation of the second kind [20]. Also, Modified analysis method for solving the Volterra integral equation of the second kind using Maple is discussed in [21].

In this article we have applied the Adomian decomposition method used by using the Maple algorithm by applying this algorithm to different examples, including finding the approximate solution and then comparing it to the exact solution and finding out the amount of error between the approximate solution and the exact solution.

The main objective of this work is to use the Adomian decomposition method in solving the Volterra-Fredholm integral equation of the second kind using Maple18.

The paper is arranged as follows: In Section 2, the Adomian decomposition method; in Section 3, numerical examples are also considered to show the ability of the proposed method, and the conclusion is drawn in Section 4.

\section{The Adomian Decomposition Method}

To clarify the basic idea of this method, we consider the following general non-linear differential equation:

$$
u(x)=f(x)+\lambda_{1} \int_{a}^{x} K_{1}(x, t) u(t) \mathrm{d} t+\lambda_{2} \int_{a}^{b} K_{2}(x, t) u(t) \mathrm{d} t .
$$

where $L$ is assumed invertible and $L^{-1}$ is an inverse operator.

The standard Adomian method defines the solution $u(x)$ by the series

$$
u(x)=\sum_{n=0}^{\infty} u_{n}(x) .
$$

The modified decomposition method

$$
u_{0}(x)=f(x)
$$




$$
\begin{gathered}
u_{1}(x)=f(x)+L^{-1}\left(\lambda_{1} \int_{a}^{x} K_{1}(x, t) u_{0}(t) \mathrm{d} t\right)+L^{-1}\left(\lambda_{2} \int_{a}^{b} K_{2}(x, t) u_{0}(t) \mathrm{d} t\right), \\
u_{n+1}(x)=L^{-1}\left(\lambda_{1} \int_{a}^{x} K_{1}(x, t) u_{n}(t) \mathrm{d} t\right)+L^{-1}\left(\lambda_{2} \int_{a}^{b} K_{2}(x, t) u_{n}(t) \mathrm{d} t\right) .
\end{gathered}
$$

The use of a modified decomposition method not only reduces the calculations but avoids the use of the human polynomial arrangement of boundaries in such cases.

\section{Numerical Examples}

In this section, we solve some examples, and we can compare the numerical results with the exact solution.

Example 1. Consider the Volterra Fredholm integral equation

$$
u(x)=x-\frac{1}{3} x^{3}+\int_{0}^{x} t u(t) \mathrm{d} t+\int_{-1}^{1} t^{2} u(t) \mathrm{d} t
$$

the exact Solution $u(x)=x$.

Applying the Adomian decomposition method using Maple18 we find (Table 1 \& Figure 1).

Example 2. Consider the Volterra Fredholm integral equation

$$
u(x)=\sin (x)-\cos (x)-\int_{0}^{x} u(t) \mathrm{d} t+\int_{0}^{\frac{\pi}{2}} u(t) \mathrm{d} t
$$

the exact Solution $u(x)=\sin (x)$.

Applying the Adomian Decomposition Method using Maple18 we find (Table 2 \& Figure 2).

Example 3. Consider the Volterra Fredholm integral equation

$$
u(x)=3 x+4 x^{2}-x^{3}-x^{4}-2+\int_{0}^{x} t u(t) \mathrm{d} t+\int_{-1}^{1} t u(t) \mathrm{d} t .
$$

the exact Solution $u(x)=3 x+4 x^{2}$.

Table 1. Approximation solution and exact solution of Volterra Fredholm integral equations for example 1.

\begin{tabular}{cccc}
\hline$x$ & $u=x$ & Exact $=x$ & Error $=\mid$ Exact1 $-u \mid$ \\
\hline 0.10000 & 0.1000000 & 0.1000000 & 0.0000000 \\
0.20000 & 0.2000000 & 0.2000000 & 0.0000000 \\
0.30000 & 0.3000000 & 0.3000000 & 0.0000000 \\
0.40000 & 0.4000000 & 0.4000000 & 0.0000000 \\
0.50000 & 0.5000000 & 0.5000000 & 0.0000000 \\
0.60000 & 0.6000000 & 0.6000000 & 0.0000000 \\
0.70000 & 0.7000000 & 0.7000000 & 0.0000000 \\
0.80000 & 0.8000000 & 0.8000000 & 0.0000000 \\
0.90000 & 0.9000000 & 0.9000000 & 0.0000000 \\
1.00000 & 1.0000000 & 1.0000000 & 0.0000000
\end{tabular}




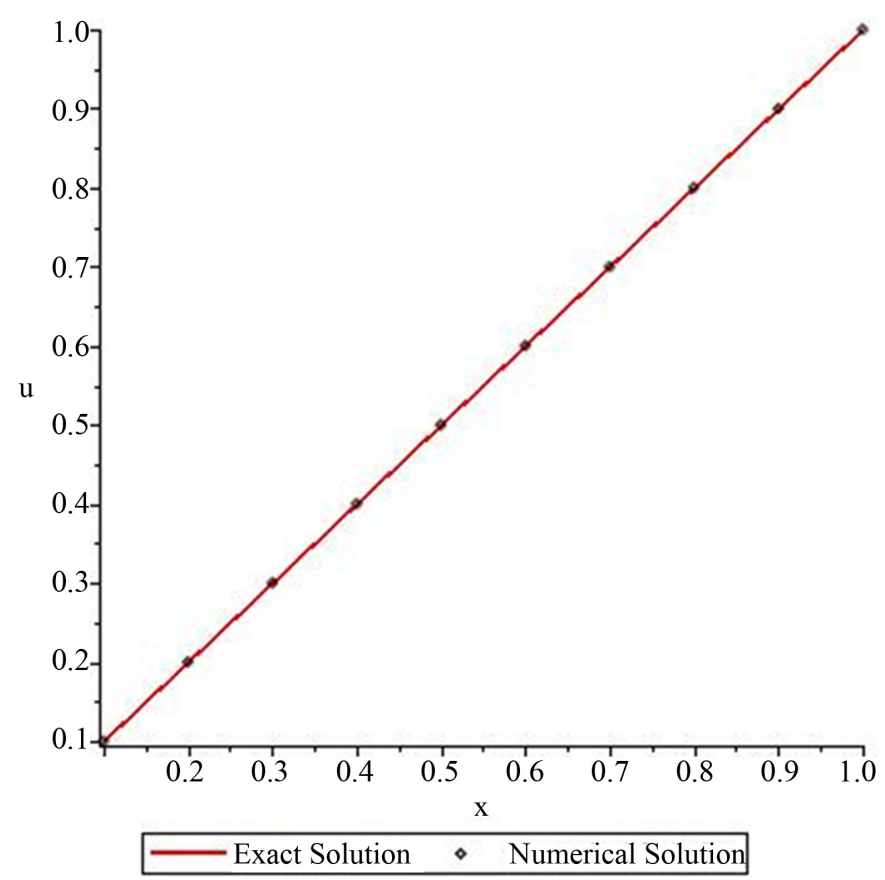

Figure 1. Plot of the solutions of Volterra Fredholm integral equation for example 1.

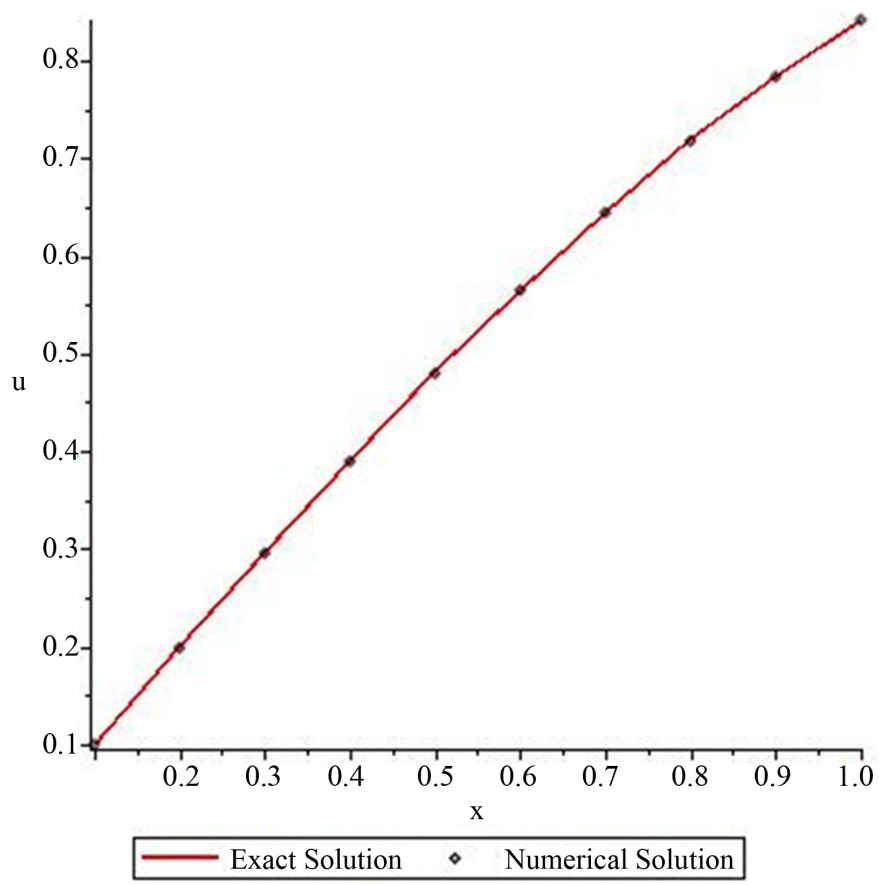

Figure 2. Plot of the solutions of Volterra Fredholm integral equation for example 2.

Table 2. Approximation solution and exact solution of Volterra Fredholm integral equations for example 2.

\begin{tabular}{cccc}
\hline$x$ & $u$ & Exact2 $=\sin (x)$ & Error $=\mid$ Exact2 $-u \mid$ \\
\hline 0.10000 & 0.0997464 & 0.0998334 & 0.0000870 \\
0.20000 & 0.1986230 & 0.1986693 & 0.0000463
\end{tabular}




\section{Continued}

\begin{tabular}{llll}
\hline 0.30000 & 0.2954967 & 0.2955202 & 0.0000235 \\
0.40000 & 0.3894071 & 0.3894183 & 0.0000113 \\
0.50000 & 0.4794205 & 0.4794255 & 0.0000050 \\
0.60000 & 0.5646404 & 0.5646425 & 0.0000021 \\
0.70000 & 0.6442169 & 0.6442177 & 0.0000008 \\
0.80000 & 0.7173558 & 0.7173561 & 0.0000003 \\
0.90000 & 0.7833268 & 0.7833269 & 0.0000001 \\
1.00000 & 0.8414710 & 0.8414710 & 0.0000000 \\
\hline
\end{tabular}

Table 3. Approximation solution and exact solution of Volterra Fredholm integral equations for example 3.

\begin{tabular}{cccc}
\hline$x$ & $u$ & Exact3 $=3 x+4 x^{2}$ & Error $=\mid$ Exact3 $-u \mid$ \\
\hline 0.10000 & 0.3400000 & 0.3399998 & 0.0000002 \\
0.20000 & 0.7600000 & 0.7599998 & 0.0000002 \\
0.30000 & 1.2600000 & 1.2599996 & 0.0000004 \\
0.40000 & 1.8400000 & 1.8399994 & 0.0000006 \\
0.50000 & 2.5000000 & 2.4999988 & 0.0000012 \\
0.60000 & 3.2400000 & 3.2399976 & 0.0000024 \\
0.70000 & 4.0600000 & 4.0599948 & 0.0000052 \\
0.80000 & 4.9600000 & 4.9599883 & 0.0000117 \\
0.90000 & 5.9400000 & 5.9399731 & 0.0000269 \\
1.00000 & 7.0000000 & 6.9999375 & 0.0000625
\end{tabular}

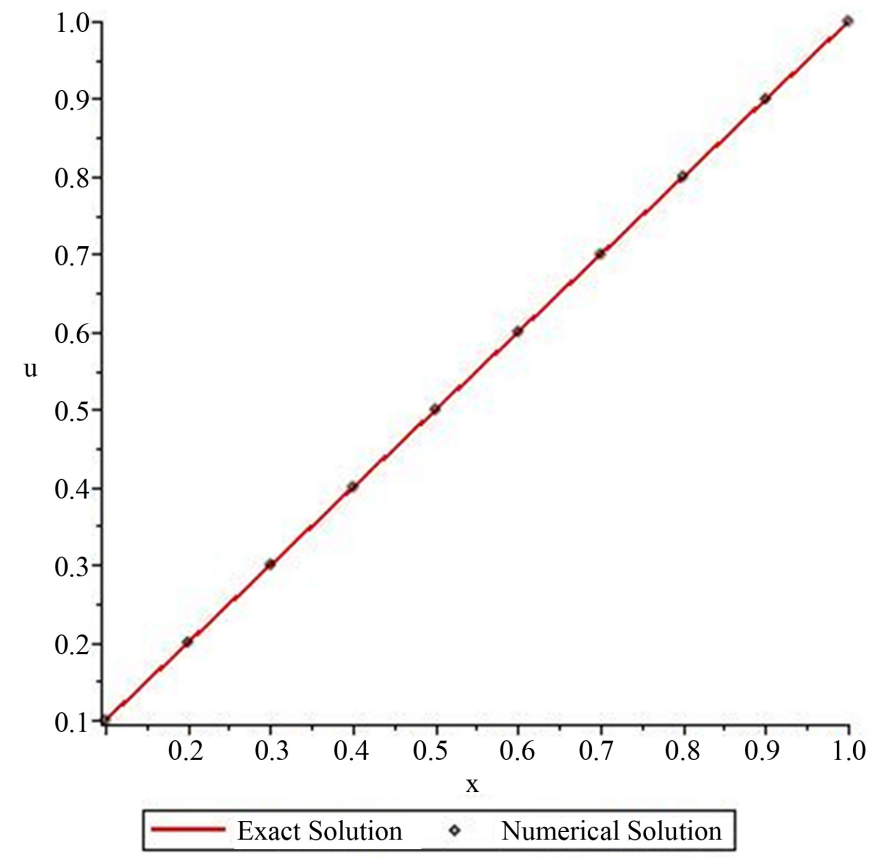

Figure 3. Plot of the solutions of Volterra Fredholm integral equation for example 3. 
Applying the Adomian Decomposition Method using Maple18 we find (Table 3 \& Figure 3).

Example 4. Consider the Volterra Fredholm integral equation

$$
u(x)=-2-2 x+2 \mathrm{e}^{x}+\int_{0}^{x}(x-t) u(t) \mathrm{d} t+\int_{0}^{1} x u(t) \mathrm{d} t
$$

the exact Solution $u(x)=x \mathrm{e}^{x}$.

Applying the Adomian Decomposition Method using Maple we find (Table 4 \& Figure 4).

Example 5. Consider the Volterra Fredholm integral equation

$$
u(x)=x^{3}-\frac{9}{20} x^{5}-\frac{1}{4} x+\frac{1}{5}+\int_{0}^{x}(x+t) u(t) \mathrm{d} t+\int_{0}^{1}(x-t) u(t) \mathrm{d} t
$$

the exact Solution $u(x)=x^{3}$.

Table 4. Approximation solution and exact solution of Volterra Fredholm integral equations for example 4.

\begin{tabular}{cccc}
\hline$x$ & $u$ & Exact $4=x \mathrm{e}^{x}$ & Error $=\mid$ Exact $4-u \mid$ \\
\hline 0.10000 & 0.1105171 & 0.1093187 & 0.0011984 \\
0.20000 & 0.2442806 & 0.2418630 & 0.0024176 \\
0.30000 & 0.4049576 & 0.4012789 & 0.0036788 \\
0.40000 & 0.5967299 & 0.5917260 & 0.0050039 \\
0.50000 & 0.8243606 & 0.8179448 & 0.0064158 \\
0.60000 & 1.0932713 & 1.0853320 & 0.0079393 \\
0.70000 & 1.4096269 & 1.4000262 & 0.0096006 \\
0.80000 & 1.7804327 & 1.7690040 & 0.0114287 \\
0.90000 & 2.2136428 & 2.2001875 & 0.0134553 \\
1.00000 & 2.7182818 & 2.7025662 & 0.0157157
\end{tabular}

Table 5. Approximation solution and exact solution of Volterra Fredholm integral equations for example 5 .

\begin{tabular}{cccc}
\hline$x$ & $u$ & Exact5 $=x^{3}$ & Error $=\mid$ Exact5 $-u \mid$ \\
\hline 0.10000 & 0.0010000 & 0.0007782 & 0.0002218 \\
0.20000 & 0.0080000 & 0.0083499 & 0.0003499 \\
0.30000 & 0.0270000 & 0.0280883 & 0.0010883 \\
0.40000 & 0.0640000 & 0.0659947 & 0.0019947 \\
0.50000 & 0.1250000 & 0.1280596 & 0.0030596 \\
0.60000 & 0.2160000 & 0.2202426 & 0.0042426 \\
0.70000 & 0.3430000 & 0.3484413 & 0.0054413 \\
0.80000 & 0.5120000 & 0.5184507 & 0.0064507 \\
0.90000 & 0.7290000 & 0.7359143 & 0.0069143 \\
1.00000 & 1.0000000 & 1.0062804 & 0.0062804 \\
\hline
\end{tabular}




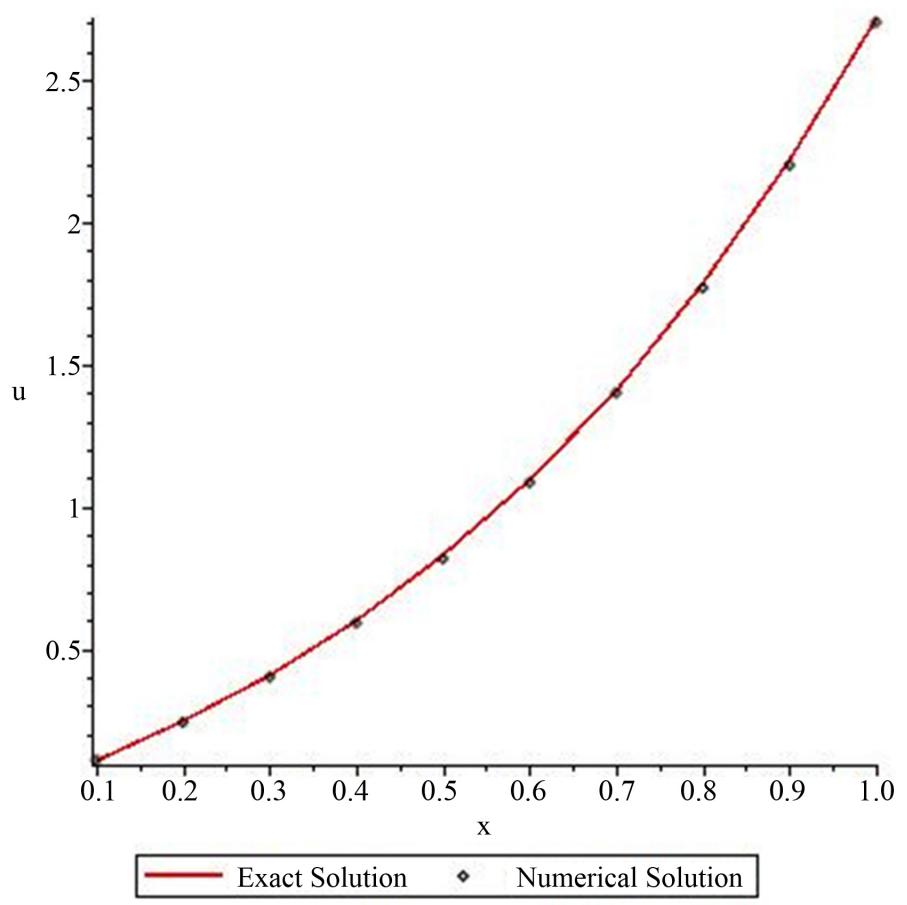

Figure 4. Plot of the solutions of Volterra Fredholm integral equation for example 4.

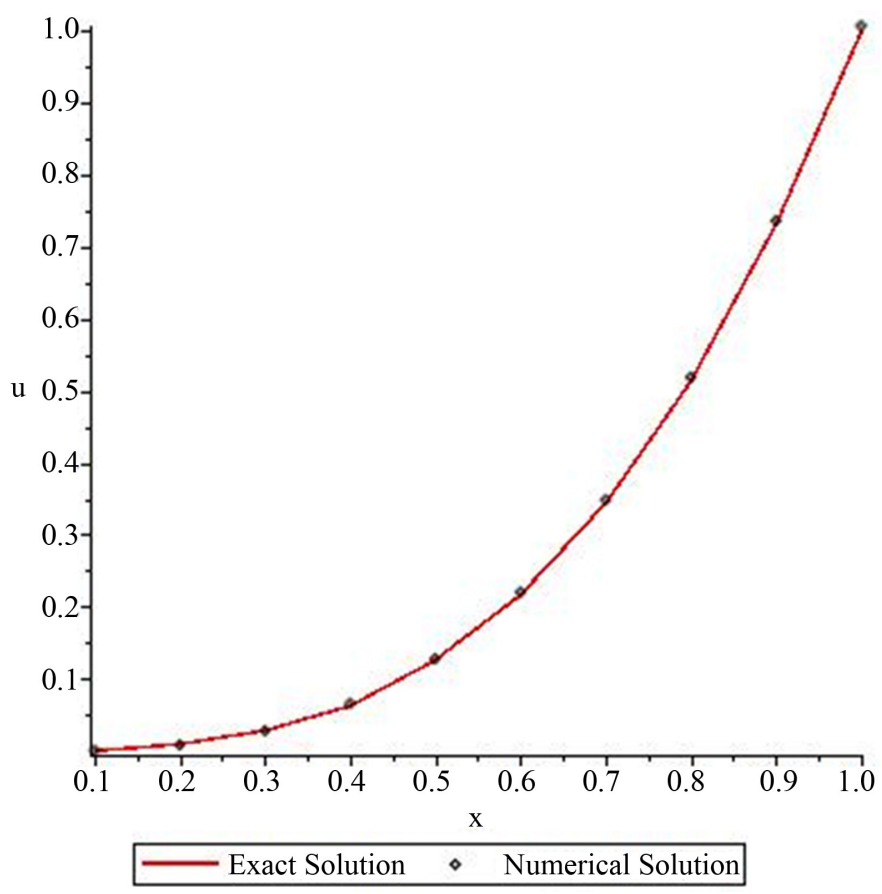

Figure 5. Plot of the solutions of Volterra Fredholm integral equation for example 5.

Applying the Adomian Decomposition Method using Maple18 we find (Table 5 \& Figure 5).

\section{Conclusion}

In this paper, the Adomian decomposition method was applied to solve the 
integral Volterra Fredholm equation using program Maple18. The results are obtained in the tables and drawn in the figures. Tables 1-5 show the correct solution and the numerical solution. Tables 1-5 represent the exact and numerical results of the examples in this article. Figures 1-5 readily show the comparison of exact solution and ap-proximate solution. Comparing the numerical results, we find that the numerical solution is largely applied to the exact solution, which proves the efficiency of the method used and the ability to obtain the numerical solution corresponding to the exact solution easily and conveniently with a program Maple 18. Moreover, the high accuracy of the results is obtained.

\section{Acknowledgements}

This project was funded by the Deanship of Scientific Research (DSR), King Abdulaziz University. The author, therefore, acknowledges with thanks for DSR technical and fnancial support.

\section{Conflicts of Interest}

The author declares no conflicts of interest regarding the publication of this paper.

\section{References}

[1] Abdul-Majid, W. (1999) A Reliable Modification of Adomain Decomposition Method. Applied Mathematics and Computation, 102, 77-86.

https://doi.org/10.1016/S0096-3003(98)10024-3

[2] Wazwaz, A.-M. and El-Sayed, S.M. (2001) A New Modification of the Adomain Decomposition Method for Linear and Nonlinear Operators. Applied Mathematics and Computation, 122, 393-405. https://doi.org/10.1016/S0096-3003(00)00060-6

[3] Vadasz, P. and Olek, S. (2000) Convergence and Accuracy of Adomian's Decomposition Method for the Solution of Lorenz Equations. International Journal of Heat and Mass Transfer, 43, 1715-1734. https://doi.org/10.1016/S0017-9310(99)00260-4

[4] El-Tawil, M.A., Bahnasawi, A.A. and Abdel-Naby, A. (2004) Solving Riccati Differential Equation Using Adomian's Decomposition Method. Applied Mathematics and Computation, 157, 503-514. https://doi.org/10.1016/j.amc.2003.08.049

[5] Ghosh, S., Roy, A. and Roy, D. (2007) An Adaptation of Adomian Decomposition for Numeric-Analytic Integration of Strongly Nonlinear and Chaotic Oscillators. Computer Methods in Applied Mechanics and Engineering, 196, 1133-1153. https://doi.org/10.1016/j.cma.2006.08.010

[6] Ebadi, G. and Rashedi, S. (2010) The Extended Adomian Decomposition Method for Fourth Order Boundary Value Problems. Acta Universitatis Apulensis, 22, 65-78.

[7] Tatari, M. and Dehghan, M. (2006) The Use of the Adomian Decomposition Method for Solving Multipoint Boundary Value Problems. Physica Scripta, 73, 672-676. https://doi.org/10.1088/0031-8949/73/6/023

[8] Azreg-Ainou, M. (2009) A Developed New Algorithm for Evaluating Adomian Polynomials. CMES Computer Modeling in Engineering and Sciences, 42, 1-18.

[9] Duan, J.S. (2010) An Efficient Algorithm for the Multivariable Adomian Polynomials. Applied Mathematics and Computation, 217, 2456-2467. 
https://doi.org/10.1016/j.amc.2010.07.046

[10] Duan, J.S. (2011) Convenient Analytic Recurrence Algorithms for the Adomian Polynomials. Applied Mathematics and Computation, 217, 6337-6348. https://doi.org/10.1016/j.amc.2011.01.007

[11] Duan, J.S., Rach, R., Baleanu, D. and Wazwaz, A.M. (2012) A Review of the Adomian Decomposition Method and Its Applications to Fractional Differential Equations. Communications in Fractional Calculus, 3, 73-99.

[12] Rach, R. (2012) A Bibliography of the Theory and Applications of the Adomian Decomposition Method, 1961-2011. Kybernetes, 41, 1087-1148. https://doi.org/10.1108/k.2012.06741gaa.007

[13] Abdul-Majid, W. (2011) Linear and Nonlinear Integral Equations. Higher Education Press, Beijing.

[14] Peter, L. (1985) Analytical and Numerical Methods for Volterra Equations. Studies in Applied Mathematics 7, SIAM, Philadelphia.

[15] Doddrell, D.M., Forbes, L.K. and Crozier, S. (1997) Calculating Current Densities and Fields Produced by Shielded Magnetic Resonance Imaging Probes. SIAM Journal on Applied Mathematics, 57, 401-425. https://doi.org/10.1137/S0036139995283110

[16] Dalal, A.M., Amani, Z.B. and Badreeh, M.G. (2014) Numerical Solution of Volterra Integral Equation of Second Kind Using Implicit Trapezoidal. Journal of Advances in Mathematics, 8, 1540-1553.

[17] Dalal, A.M. (2014) Adomian Decomposition Method of Fredholm Integral Equation of the Second Kind Using Maple. Journal of Advances in Mathematics, 9, 1868-1875.

[18] Dalal, A.M. (2014) Application of Adomian Decomposition Method for Solving of Fredholm Integral Equation of the Second Kind. European Journal of Science and Engineering, 9, 1-9.

[19] Dalal, A.M. (2016) Adomian Decomposition Method for Solving of Fredholm Integral Equation of the Second Kind Using Matlab. International Journal of GEOMATE, 11, 2830-2833.

[20] Dalal, A.M. and Honida, M.M. (2018) Numerical Solution of System of Three Nonlinear Volterra Integral Equation Using Implicit Trapezoidal. Journal of Mathematics Research, 10, 44. https://doi.org/10.5539/jmr.v10n1p44

[21] Dalal, A.M. (2019) The Modified Decomposition Method for Solving Volterra Integral Equation of the Second Kind Using Maple. International Journal of GEOMATE, 17, 23-28. https://doi.org/10.21660/2019.62.4590 